\title{
The need for individual testing of applications aimed at early detection of skin cancer
}

\author{
Jelle Stans \\ Institute for Globally Distributed Open Research and Education, Beringen, Belgium
}

The increasing use of smartphones and technological advances has given rise to a range of applications for medical and healthcare purposes. The field of oncology is no exception, with applications being made available for, amongst others, education, treatment information, prevention and early detection [1]. A large proportion of the applications claiming to provide the opportunity for early detection are active within the field of skin cancer.

Several types of apps are available in the area of skin cancer. Some apps provide general information about melanoma, others provide information about prevention and a number of apps provide an estimate of the risk whether a certain lesion is malignant based on a picture taken [2]. A systematic review conducted in 2018 showed that the accuracy of the latter type of app was not sufficient at that time [3]. The evidence was also rather limited in quality and quantity. This was confirmed by a very recent systematic review [4] which showed that apps currently are unable to identify all skin cancers. In this case, the studies were also of limited methodological quality and had relatively small sample sizes. In another review [5], the sensitivity ranged from $7 \%$ to $87 \%$. Another problem that was often mentioned, is the high rate of unusable images taken $[2,3,5]$.

Due to the variation in accuracy between apps, it is important that every app intended for medical use is individually tested for accuracy. This could be set as a requirement for regulatory approval. Currently this is not always the case when applying for a CE-mark. An example of an application that is aiming to become part of medical care is SkinVision (SkinVision, Amsterdam) In the Benelux, it seems that this app has gone the furthest in the direction of regulatory approval. It is included in the Belgian mHealth validation pyramid and has received a CE-mark as a class I medical device. It is additionally cooperating with a Dutch health insurer. However, the app has also attracted criticism from the Dutch association of dermatologists.

In general, several types of skin cancer applications can provide useful information for users. However, it seems that more research is needed to allow for applications that provide enough sensitivity and specificity for routine medical use and self-screening. Each application that is used for such purposes should be independently assessed using an adequate study design before by being utilised. By making these results public, they can be assessed by the different stakeholders.

Confilct of interest: none declared

\section{Jelle Stans}

Institute for Globally Distributed Open Research and Education

Beringen, Belgium

e-mail:jelle.stans@igdore.org

Received: 9 Sep 2020

Accepted: 17 Sep 2020

\section{References}

1. Charbonneau D, Hightower S, Katz A, et al. Smartphone apps for cancer: A content analysis of the digital health marketplace. Digit Health. 2020; 6: 205520762090541, doi: 10.1177/2055207620905413.

2. Chao E, Meenan C, Ferris L. Smartphone-Based Applications for Skin Monitoring and Melanoma Detection. Dermatol Clin. 2017; 35(4): 551-557, doi: 10.1016/j.det.2017.06.014.

\section{How to cite:}

Stans J. The need for individual testing of applications aimed at early detection of skin cancer. NOWOTWORY J Oncol 2020; 70: 276-277. 
3. Chuchu N, Takwoingi Y, Dinnes J, et al. Smartphone applications for triaging adults with skin lesions that are suspicious for melanoma. Cochrane Database Syst Rev. 2018, doi: 10.1002/14651858.cd013192.

4. Freeman K, Dinnes J, Chuchu N, et al. Algorithm based smartphone apps to assess risk of skin cancer in adults: systematic review of diagnostic accuracy studies. BMJ. 2020: m127, doi: 10.1136/bmj.m127.
5. Rat C, Hild S, Sérandour JR, et al. Use of Smartphones for Early Detection of Melanoma: Systematic Review. J Med Internet Res. 2018; 20(4): e135, doi: 10.2196/jmir.9392. 